\title{
Sensitivity of three antibody assays to SARS-CoV-2 nucleocapsid protein in relation to timing since diagnosis
}

\author{
Tetsuya Mizoue ${ }^{1, *}$, Shohei Yamamoto', Akihito Tanaka², Yusuke Oshiro², Natsumi Inamura², Maki Konishi', \\ Mitsuru Ozeki ${ }^{2}$, Norio Ohmagari ${ }^{3}$ \\ ${ }^{1}$ Department of Epidemiology and Prevention, Center for Clinical Sciences, National Center for Global Health and Medicine, Toyko, Japan; \\ ${ }^{2}$ Department of Laboratory Testing, Center Hospital of the National Center for the Global Health and Medicine, Tokyo, Japan; \\ ${ }^{3}$ Disease Control and Prevention Center, National Center for Global Health and Medicine, Tokyo, Japan.
}

\begin{abstract}
The sensitivity of immunoassay for antibodies to SARS-CoV-2 may decrease over time. We compared three serology assays against SARS-CoV-2 nucleocapsid protein using serum samples of 20 health care workers with a history of mild PCR-confirmed coronavirus disease 2019 (COVID-19) at various timing since diagnosis. Overall, the sensitivity (95\% confidence interval) was 95.0 (75.1-99.9), 60.0 (36.1-81.0), and 45.0 (23.1-68.5) for the Roche, Sysmex, and Abbott assays, respectively. The sensitivity of all these assays exceeded 80 for those diagnosed within 6 months, whereas it varied considerably for those diagnosed more than 6 months ago: 88.9 (Roche) versus 0 (Abbott). The present data provide a reference for researchers planning serological studies and interpreting these data. Such assay difference in terms of detection durability can be used, if used in combination, for the estimation of the timing of previously undetected infection.
\end{abstract}

Keywords: COVID-19, SARS-CoV-2, immunoassay, antibody, sero-epidemiologic studies, Japan

\section{Introduction}

Epidemiological data on the spread of SARS-CoV-2 infection are helpful for planning strategies to control coronavirus disease 2019 (COVID-19). The number of COVID-19 cases confirmed by reverse-transcription polymerase chain reaction (RT-PCR), which has been performed mainly for symptomatic cases, does not represent the whole picture of this infection. In this context, serological study has been performed to assess the undiagnosed infection of SARS-CoV-2 in a population (1).

Several immunoassays for antibodies to SARSCoV-2 have been developed and proved to be highly sensitive in detecting recently diagnosed cases (2). Due to the waning of antibodies within months, especially among those without symptoms (3), however, a concern has been raised about the increasing gap between seroprevalence and cumulative infection over time.

Following an earlier report that showed a variation in sensitivity among assays after 3-4 months since disease onset (4), several serological studies with a longer period of follow-up have demonstrated an even larger between-assay variation in sensitivity (5-7). Data on this issue is scarce in Asians, who recorded higher maximum anti-nucleocapsid levels after infection (3). Here we compared three immunoassays against SARS-
CoV-2 nucleocapsid protein among health care workers with a history of COVID-19 in a Japanese hospital.

\section{Sero-epidemiological study among health care workers}

A repeat serological study has been conducted since July 2020 among workers of National Center for Global Health and Medicine (NCGM) (8), which has accepted many patients with severe COVID-19. We asked participants about COVID-19 related information including a history of COVID-19 and the date of diagnosis, which were confirmed against records kept by the infection control department. The current study included participants who reported a history of COVID-19 at the third survey (June 2021). Written informed consent was obtained from each participant, and the study procedure was approved by the ethics committee of NCGM.

\section{Assessment of three antibody assays}

We measured antibodies against SARS-CoV-2 nucleocapsid protein, which increased after SARSCoV-2 infection but not after vaccination, using three commercially available kits; namely, Elecsys ${ }^{\circledR}$ AntiSARS-CoV-2 immunoassay (Roche diagnostic), 

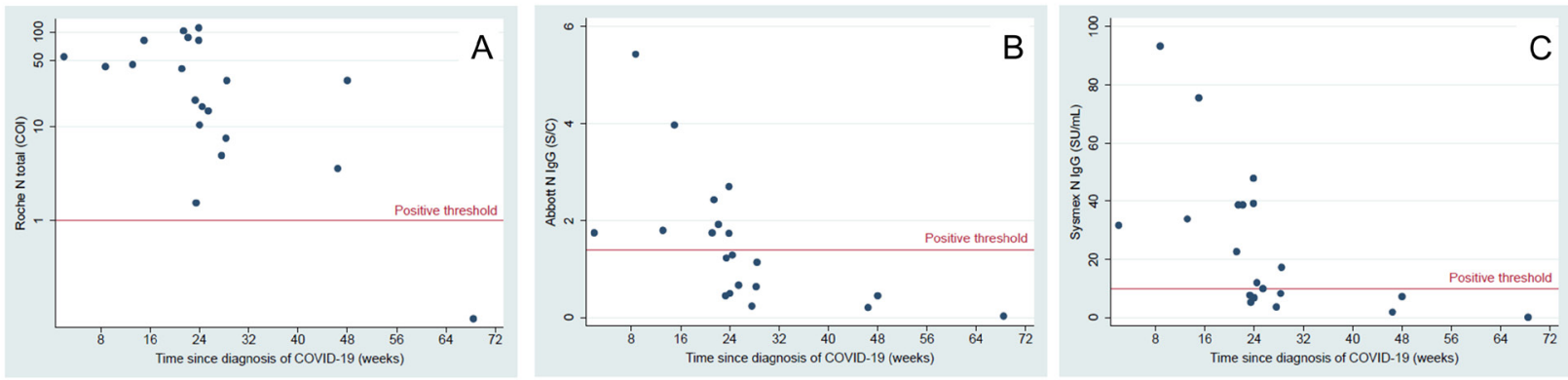

Figure 1. SARS-CoV-2 antibody titers of the Roche (A), Abbott (B), and Sysmex (C) assays with time since diagnosis of COVID-19.

Table 1. Sensitivity of three SARS-CoV-2 antibody assays according to time since diagnosis of COVID-19

\begin{tabular}{|c|c|c|c|c|}
\hline \multirow{3}{*}{ Items } & \multicolumn{4}{|c|}{ Timing of COVID-19 diagnosis with RT-PCR } \\
\hline & \multicolumn{2}{|c|}{ Over 6 months ( 24 weeks) ago } & \multicolumn{2}{|c|}{ Within 6 months (24 weeks) } \\
\hline & No. of positives / Total number & Sensitivity $(95 \% \mathrm{CI})$ & No. of positives /Total number & Sensitivity $(95 \% \mathrm{CI})$ \\
\hline Roche & $8 / 9$ & $88.9(51.8-99.7)$ & $11 / 11$ & $100(71.5-100)$ \\
\hline Sysmex & $3 / 9$ & $33.3(7.5-70.1)$ & $9 / 11$ & $81.8(48.2-97.7)$ \\
\hline Abbott & $0 / 9$ & $0(0-33.6)$ & $9 / 11$ & $81.8(48.2-97.7)$ \\
\hline
\end{tabular}

Abbreviations: CI, confidence interval; COVID-19, coronavirus disease 2019; RT-PCR, reverse transcription-polymerase chain reaction; SARS-

$\mathrm{CoV}-2$, severe acute respiratory syndrome coronavirus 2 .

SARS-CoV-2 IgG assay (Abbott), and HISCL antiSARS-CoV-2 immunoassay (Sysmex). We plotted immunoglobulin titer/assay signal against time since COVID-19 diagnosis and calculated the proportion of detecting COVID-19 confirmed cases (sensitivity) for each assay according to time since diagnosis.

\section{Identification of individuals with a history of COVID-19}

Of 2,763 participants ( $89 \%$ ) of the third survey, 20 selfreported a history of RT-PCR confirmed COVID-19, which was confirmed against the hospital record. Of these, $5(25 \%)$ were admitted to a hospital while contracting COVID-19, but none received mechanical ventilation; $14(70 \%)$ received Pfizer-BioNTech vaccine twice.

\section{Comparison of seropositive rate according to the timing since diagnosis}

In the Roche assay, 19 of 20 previously diagnosed COVID-19 cases showed positive, and the titer appears not to be related to the timing of diagnosis (Figure 1A). In the Abbott assay, only 9 of 20 cases were positive, and the proportion of positive cases dramatically decreased over time, with all 9 cases diagnosed more than 6 months ago being negative (Figure 1B). The Sysmex assay also showed a decreasing trend of positivity over time; 12 of 20 cases were seropositive, and two-thirds of cases diagnosed more than 6 months ago showed negative $(n=6 / 9)$ (Figure 1C).

\section{Comparison of sensitivity according to the timing} since diagnosis

Overall, the sensitivity ( $95 \%$ confidence interval) was 95.0 (75.1-99.9), 60.0 (36.1-81.0), and 45.0 (23.1-68.5) for the Roche, Sysmex, and Abbott assays, respectively. The sensitivity of these assays exceeded 80 for those diagnosed within 6 months, whereas it varied considerably for those diagnosed more than 6 months ago: 88.9 (Roche) versus 0 (Abbott) (Table 1).

Careful evaluation of assay is needed when planning serological studies and interpreting data in the later stages of a pandemic

In a cross-sectional analysis of health care staff with a history of COVID-19, we found a remarkable difference in the sensitivity over time among the three commercially available assays, with sensitivity being the highest in the Roche assay, followed by the Sysmex and Abbott assays.

The three assays showed a sensitivity of over 80 for the samples taken from patients who were diagnosed within 6 months. However, the Abbott and Sysmex assays, both of which are targeted for IgG SARS-CoV-2 nucleocapsid protein, showed a considerably low sensitivity for the samples taken more than 6 months after diagnosis. Similarly, long-term repeat serological studies have reported a high sensitivity of the Roche 
assay but a considerably low sensitivity of the Abbott assay toward the end of follow-up (5-7).

The population studied was comprised of individuals with a history of mild COVID-19, which has been associated with lower antibody titers than severe one (6). Given that mild cases explain a major portion of this disease, the large between-assay difference in sensitivity observed in the present as well as previous studies highlights the need for careful evaluation of assay when planning serological studies and interpreting data in the later period of the pandemic.

We should acknowledge study limitations. First, due to the small size of the study $(n=20)$, the estimated sensitivities have wide confidence intervals. Second, as mentioned above, our study included only patients with mild COVID-19. The result may not be applied to severe or asymptomatic cases. Third, the crosssectional design of the study limits our inference about the change of antibody status over time for each case.

\section{Conclusions and future directions}

The present data provide a reference for researchers planning serological studies and interpreting these data. Further, such large difference among assays in terms of detection durability can be used, if used in combination, for the estimation of the timing of previously undetected infection. More research is required to examine whether the use of multiple assays can help differentiate the impact of the recent epidemic from earlier ones.

\section{Acknowledgements}

We thank Haruka Osawa for her contribution to data collection.

Funding: This work was funded by the NCGM COVID-19 Gift Fund (19K059) and the Japan Health Research Promotion Bureau Research Fund (2020-B$09)$. Antibody assay reagents of Elecsys ${ }^{\circledR}$ Anti-SARSCoV-2 immunoassay and SARS-CoV-2 IgG assay were provided by Abbott Japan and Roche Diagnostic, respectively.

Conflict of Interest: The authors have no conflicts of interest to disclose.

\section{References}

1. Kalish H, Klumpp-Thomas C, Hunsberger S, et al. Undiagnosed SARS-CoV-2 seropositivity during the first 6 months of the COVID-19 pandemic in the United States. Sci Transl Med. 2021; 13:eabh3826.

2. National SARS-CoV-2 Serology Assay Evaluation Group. Performance characteristics of five immunoassays for SARS-CoV-2: a head-to-head benchmark comparison. Lancet Infect Dis. 2020; 20:1390-1400.

3. Lumley SF, Wei J, O'Donnell D, et al. The duration, dynamics, and determinants of severe acute respiratory syndrome coronavirus 2 (SARS-CoV-2) antibody responses in individual healthcare workers. Clin Infect Dis. 2021; 73:e699-e 709.

4. Muecksch F, Wise H, Batchelor B, et al. Longitudinal serological analysis and neutralizing antibody levels in coronavirus disease 2019 convalescent patients. J Infect Dis. 2021; 223:389-398.

5. Schallier A, De Baets S, De Bruyne D, Dauwe K, Herpol M, Couck P. Assay dependence of long-term kinetics of SARS-CoV-2 antibodies. Diagn Microbiol Infect Dis. 2021; 100:115403.

6. Peluso MJ, Takahashi S, Hakim J, et al. SARS-CoV-2 antibody magnitude and detectability are driven by disease severity, timing, and assay. Sci Adv. 2021; 7:eabh3409.

7. Kerr C, Allen N, Hughes G, Kelly M, O'Rourke F, Lynagh Y, Dunne J, Crowley B, Conlon N, Bergin C. SARS-CoV-2 anti-nucleocapsid assay performance in healthcare workers at baseline and 6 months. Ir J Med Sci. 2021; doi: 10.1007/s11845-021-02700-5.

8. Yamamoto S, Tanaka A, Oshiro Y, Ishii M, Ishiwari H, Konishi M, Matsuda K, Ozeki M, Miyo K, Maeda K, Mizoue T, Sugiura W, Mitsuya H, Sugiyama H, Ohmagari N. Seroprevalence of SARS-CoV-2 antibodies in a national hospital and affiliated facility after the second epidemic wave of Japan. J Infect. 2021; 83:237279.

Received October 12, 2021; Revised November 1, 2021; Accepted November 25, 2021.

Released online in J-STAGE as advance publication November 30, 2021.

*Address correspondence to:

Tetsuya Mizoue, Department of Epidemiology and Prevention, National Center for Global Health and Medicine, 1-21-1, Toyama, Shinjuku-ku, Tokyo 162-8655, Japan.

E-mail: mizoue@hosp.ncgm.go.jp 